\title{
Assessing Clinical Change in Individuals Exposed to Repetitive Head Impacts: The Repetitive Head Impact Composite Index
}

Charles Bernick ( $\nabla$ bernick@uw.edu )

Cleveland Clinic 800 W. Bonneville Ave Las Vegas, NV 89104 https://orcid.org/0000-0003-4040-3587

\section{Guogen Shan}

University of Nevada Las Vegas, Las Vegas 4505 S. Maryland Parkway Las Vegas, NV 89154

\section{Lauren Bennett}

Cleveland Clinic 800 W. Bonneville Ave Las Vegas, NV 89104

\section{Jay Alberts}

Cleveland Clinic 9500 Euclid Ave Cleveland, OH 44195

\section{Jeffrey Cummings}

University of Nevada Las Vegas, Las Vegas 4505 S. Maryland Parkway Las Vegas, NV 89154

\section{Research}

Keywords: traumatic brain injury, chronic traumatic encephalopathy, cognitive measures

Posted Date: August 10th, 2020

DOl: https://doi.org/10.21203/rs.3.rs-52183/v1

License: (c) (i) This work is licensed under a Creative Commons Attribution 4.0 International License. Read Full License 


\section{Abstract}

Background: There is a current lack of any composite measure for the effective tracking and monitoring of executive function in individuals exposed to repetitive head impacts (RHI). The aim of this study is to create a composite instrument for the purposes of detecting change over time in executive function in individuals exposed to $\mathrm{RHI}$.

Methods: The data to derive the composite instrument came from the Professional Fighters Brain Health Study (PFBHS), a longitudinal study of active and retired professional fighters (boxers and mixed martial arts [MMA] fighters) and healthy controls. Participants in the PFBHS underwent assessment on an annual basis that included computerized cognitive testing and behavioral questionnaires. Multivariate logistic regression models were employed to compare active fighters $(n=117)$ with controls $(n=22)$, and retired fighters $(n=26)$ with controls to identify the predictors that could be used to differentiate the groups over time. In a second step, linear discriminant analysis was performed to derive the linear discriminant coefficients for the three groups by using the predictors from the two separate logistic regression models.

Results: The composite scale is a weighted linear value of 12 standardized scores consisting of both current and yearly change scores in domains including: processing speed, choice reaction time, semantic fluency, letter fluency, and Barrett Impulsiveness Scale. Because the weighting of values differed between active and retired fighters, two versions emerged. The mean and standard deviation ratio (MSDR) showed that the new index had better sensitivity compared to the individual measures, with the ratio of MSDR of the new index to that of the existing measures of at least 1.84.

Conclusion: With the increasing need for tools to follow individuals exposed to $\mathrm{RHI}$ and the potential of clinical trials on the horizon for $\mathrm{CTE}$, the $\mathrm{RHICl}$ is poised to serve as an initial approach to a composite clinical measure.

\section{Background}

There is increasing interest in understanding the long term neurological effects of exposure to repetitive head impacts (RHI) including Chronic Traumatic Encephalopathy (CTE), with the anticipation of developing therapeutic interventions (1-3). Currently, the tools we apply to track cognitive and behavioral changes in those exposed to $\mathrm{RHI}$ are borrowed from standard neuropsychological tests and behavioral instruments. We lack any composite measures that are focused on the domains that are most likely to show change due to $\mathrm{RHI}$.

To develop and validate new test batteries takes significant time and effort. Composite scales can be produced by either consensus (e.g. United Parkinson's Disease Research Scale) or data driven (e.g., Alzheimer's disease Composite Score [ADCOMS]) strategies (4-6). The data-driven approach leverages existing scales and uses mathematical approaches to identify the items most sensitive to change in the group being studied and applies weighting to improve performance. 
The aim of this study is to create a composite instrument that could detect change over time in clinical measures in individuals exposed to RHI. This was accomplished by utilizing data obtained from the Professional Fighters Brain Health Study (PFBHS), a longitudinal study of professional boxers and mixed martial arts (MMA) fighters -individuals who are either actively or previously exposed to RHI (7). The composite scale -- termed Repetitive Head Impact Composite Index (RHICl) -- if validated in prospective studies, could be used for natural history studies of RHI, clinical trials of traumatic encephalopathy syndrome (TES), or other mild traumatic brain injury research.

\section{Methods}

The data used for the $\mathrm{RHICl}$ came from the PFBHS consisting of active and retired professional fighters (boxers and MMA fighters) and healthy controls. Active fighters were required to have at least 1 professional fight within 2 years of enrollment and be training with the intent to compete. Retired fighters were included if they had been boxers, had a minimum of 10 professional fights, had no sanctioned fights for at least two years, and did not intend to return to competition (there were too few retired MMA fighters to include as a separate group). Control subjects were recruited from outreach efforts in the community and could not have any prior history of neurological disorders, head trauma, military service, or participation at a high school level or higher in a combat sport or a sport in which head impacts can be anticipated to occur, such as football, wrestling, hockey, rugby, soccer, or rodeo. All participants were required to be able to read at minimum at a $4^{\text {th }}$ grade level but were not otherwise screened for cognitive status prior to enrollment.

Enrollment in the PFBHS began in 2011 and has been continuous since. Each participant is seen on an annual basis, and for active fighters, not sooner than 45 days after a sanctioned fight. The PFBHS was approved by the Cleveland Clinic Institutional Review Board and written informed consent was obtained from all participants. More detailed methods of recruitment and study procedures have been described previously (7).

At baseline and each annual visit, a battery of tests and information were acquired including magnetic resonance imaging (MRI) of the brain, computerized cognitive testing, behavioral questionnaires and exposure history. Participants answered questionnaires with the assistance of the study coordinator that collected information on demographics; educational attainment; medical history including concurrent illnesses and prescribed medications; previous head trauma, both related and unrelated to athletic activities; and prior involvement in other contacts sports.

Cognitive function was assessed by two computerized cognitive test batteries and verbally administered measures of verbal fluency. One computerized battery consists of four subtests of the CNS Vital Signs ${ }^{\text {Tm }}$ (CNS Vital Signs ${ }^{\mathrm{TM}}$, North Carolina) including verbal memory, symbol digit coding, Stroop and a finger tapping test. CNS Vital Signs ${ }^{\text {TM }}$ offers robust and reliable measurements of cognition, which are computerized; test performance is supervised by a technician (8). Results from these tests are used to create scores in the following clinical domains: verbal memory, processing speed, psychomotor speed 
and reaction time. The other computerized cognitive assessment, C3 Logix, an iPad-based test that includes a processing speed test, Trail Making Test Parts A and B, simple and choice reaction time paradigms along with a measure of balance measure (9). Measures of verbal fluency include both letter (words that start with "f") and semantic (animals) fluency tasks.

Behavioral assessment was obtained through administration of the Patient Health Questionnaire (PHQ 9) that evaluates for the presence of depressive symptoms and the Barrett Impulsiveness Scale (BIS II)(10, 11). The BIS II comprises 30 questions, which have been shown to load on six factors (attention, cognitive instability, motor, perseverance, self-control, cognitive complexity); the items loading on these factors provide the subscales for the instrument. For both total and subscale scores, higher scores refer to higher levels of impulsiveness.

\section{Statistical methods}

\section{Samples}

Data from the PFBHS study were used to develop a new composite index for individuals exposed to RHI. We included participants having at least three study visits over a minimum of 2 years. The normal controls ( $n=22$ ) were used as the reference group with the assumption that they were the least likely to change over a several year period. The conceptual framework informing the index was to identify and create a composite from those cognitive and behavioral measures that were able to best differentiate over time the active fighters $(n=117)$ from the controls and the retired fighters $(n=26)$ from the controls.

The initial step was to examine the correlation of the cognitive and behavioral variables with each other in a correlation matrix. This was performed to avoid the problem that could be caused by multiple colinearity - when two highly correlated variables are both included in a statistical model may result in the sign change of parameter estimates. When two or more measures were highly correlated, the study team adjudicated which one was clinically more important.

After removing the highly correlated measures, 16 items from cognitive and behavioral assessments were initially included in the new index. Among these 16 measures, 12 were cognitive measures (C) and 4 were behavioral measures (B). Although we had longitudinal data from multiple time points, the change of the last measure from baseline was used to avoid the violation of the linear assumption of the longitudinal measures. For each measure, we utilized the score and the average yearly change in score based on the last measure and the baseline, comprising a total of 32 predictors.

\section{New composite index}

Multivariate logistic regression models were employed to compare active fighters with controls, and retired fighters with controls to identify the predictors that could be used to differentiate the groups. In a second step, linear discriminant analysis was performed to derive the linear discriminant coefficients for the three groups by using the predictors from the two separate logistic regression models. 
Assuming that CA and BA represent the average yearly changes of cognitive measures and behavioral measures, respectively, we standardized all the measures by using the range of each measure from all samples. The multinomial logistic regression between the active fighter group and the control group was presented as

$\mathrm{g}(\operatorname{Pr}(\mathrm{Y}=\mathrm{i}\rfloor \mathrm{X}))=\sum_{i=1}^{12} \beta_{i} C_{i}+\sum_{i=1}^{12} \gamma_{i} C A_{i}+\sum_{i=1}^{4} \varphi_{i} B_{i}+\sum_{i=1}^{4} \omega_{i} B A_{i}$,

where $g(p)=\log (p /(1-p))$ is the logit link function, $X$ are the observed data, and $Y$ is the group with 0 for the control group and 1 for the active fighter group. A similar logistic regression model was used for the retired fighter group using the control group as the reference group. We utilized the backward model selection method with the p-value of 0.3 as the threshold to select the measures that can be used in the next step in developing a new composite index. The selected predictors that did not meet the direction were removed from the final model. The final model had a total of 12 measures among the 32 predictors from cognitive and behavioral assessments.

In the final step of composite construction, we performed discriminant analysis in which the group variable (control, active fighters, and retired fighters) was used as the outcome, and the 12 measures were included in the model as independent variables. Discriminant analysis was performed to derive the linear discriminant coefficients for the 12 measures in each group. We then rescaled the new index to the range of 0 to 20 with lower scores considered to be normal.

\section{Results}

The characteristics of the participants that make up the data set are shown in Table 1. The proposed composite index is a weighted linear value of the 12 standardized scores with 6 sub-scores: processing speed, choice reaction time, semantic fluency (both total correct score and number of repeated words), letter fluency (number of repeated words), and Barrett Impulsiveness Scale Question 2 (I do things without thinking); and 6 yearly change sub-scores that capture both the baseline difference and the longitudinal change over time: choice reaction time, semantic fluency (total correct and number of repeats), letter fluency (number of repeats), Barrett Impulsiveness Scale question 2 and 6 (I have racing thoughts). Because the weighting of values differed between active and retired fighters, two versions of the $\mathrm{RHICl}$ emerged. 
Table 1

\begin{tabular}{|c|c|c|c|c|}
\hline & Active Fighters & Retired Fighters & Control & p-value \\
\hline $\mathrm{N}$ & $117(70.9 \%)$ & $26(15.8 \%)$ & $22(13.3 \%)$ & \\
\hline Age & $29.9(6.3)$ & $47.2(10.0)$ & $35.1(14.7)$ & $<0.0001$ \\
\hline Education Years & $13.5(2.0)$ & $13.7(2.1)$ & $14.8(2.8)$ & 0.0496 \\
\hline Female & $16(13.7 \%)$ & $1(3.9 \%)$ & $2(9.1 \%)$ & 0.3390 \\
\hline Number of fights & $9.6(11.3)$ & $34.3(16.6)$ & 0 & $<0.0001$ \\
\hline Years of fighting & $4.6(4.4)$ & $9.7(5.1)$ & 0 & $<0.0001$ \\
\hline Race & & & & 0.5686 \\
\hline African American & $26(22.2 \%)$ & $3(11.5 \%)$ & $3(13.6 \%)$ & \\
\hline White & $55(47.0 \%)$ & $16(61.5 \%)$ & $11(50.0 \%)$ & \\
\hline Other & $36(30.8 \%)$ & $7(26.9 \%)$ & $8(36.4 \%)$ & \\
\hline
\end{tabular}

The range of the new index was from 0 to 20 . We evaluated the performance of the new index in the active fighters as compared to the individual measures with regards to sensitivity. The sensitivity was calculated as the mean and standard deviation ratio (MSDR) over a fixed follow-up time (e.g., 2 years). A larger MSDR value represented a larger effect size, which leads to greater sensitivity.

We computed the MSDR using the 2-year data of each fighter (e.g., the 1st and 3rd visits). The MSDR (Table 2) showed that the new index had better sensitivity compared to the individual measures, with the ratio of MSDR of the new index to that of the existing measures of at least 1.84.

Table 2

\section{Mean and Standard Deviation Ratio}

\begin{tabular}{|lll|}
\hline & Active fighters (Ratio) & Retire fighters (Ratio) \\
\hline RHICl & $5.79(1.00)$ & $4.89(1.00)$ \\
\hline Processing speed score & $2.23(2.59)$ & $2.38(2.05)$ \\
\hline Choice reaction time & $1.94(2.98)$ & $2.66(1.84)$ \\
\hline Letter fluency total correct score & $1.96(2.95)$ & $1.69(2.90)$ \\
\hline $\begin{array}{l}\text { Comparison of the Mean and Standard Deviation Ratio between the Repetitive Head Impact } \\
\text { Composite Index (RHICl) and individual scores on processing speed, choice reaction time, or letter }\end{array}$ \\
\hline
\end{tabular}


We showed substantial improvement in sensitivity as compared to the individual measures. The improved sensitivity of the $\mathrm{RHICl}$ would be expected to reduce the sample size for a clinical trial if used as an outcome measure. As an example, consider a hypothetical 2-year study to detect a $5 \%$ decline from baseline for active fighters using the existing measures, to attain $90 \%$ power at the significance level of 0.05. The required sample size based on processing speed score was 126; it was reduced to 25 when the new index was used. Similar results were observed when other measures were used in sample size calculations.

\section{Discussion}

Assessment tools that are composed of elements of existing scales thought most likely to detect change in a particular disease are commonly used as outcome measures in clinical trials and observational studies (12). There are several advantages to using composite measures for clinical and research purposes including improved power to detect change (thus potentially necessitating fewer participants for clinical trials), avoiding arbitrary choices between several important outcomes that occur in the same disease, and serving as a common measure that is comparable between groups (13-14).

Though there has been increasing attention to the long-term sequelae of RHI including TES and CTE, no validated composite measure is available. The $\mathrm{RHICl}$ is a first attempt using data-driven methods to construct a composite scale to be used in tracking longitudinal change and as a potential outcome measure in studies related to neurological effects of $\mathrm{RHI}$. The $\mathrm{RHICl}$ has the advantage of easy administration on a desktop or tablet computer, with most subtests performed on the device itself or the score entered by the person supervising the testing (verbal fluency).

The components that constitute the $\mathrm{RHICl}$ currently, including measures of processing speed, attention, executive function and impulsivity, are consistent with domains that have been reported in the literature as effected in boxers and other groups exposed to RHI $(2,15-16)$. Delayed memory did not emerge as a component of the $\mathrm{RHICl}$ despite memory complaints by many with $\mathrm{RHI}$ and impaired memory function being reported in individuals who had a confirmed pathological diagnosis of CTE $(17,18)$. The lack of memory elements in the $\mathrm{RHICl}$ may reflect the predominance of executive dysfunction associated with $\mathrm{RHI}$. Alternatively, as we accumulate greater numbers of older retired fighters in our sample (or older former athletes in other samples tested), it is possible that this domain will enter the model and be added to the $\mathrm{RHICl}$.

The structure and weighting of items in the $\mathrm{RHICl}$ differed between active and retired fighters. The underlying pathophysiologic process may differ between these two groups. In the active fighter group, $\mathrm{RHICl}$ may be measuring the effects of accumulating axonal injury whereas the retired fighters may include some who are harboring a neurodegenerative process due to CTE. Longitudinal MRI regional volumetric data suggest such a dichotomy (19). The practical implication is that in implementing the $\mathrm{RHICl}$, the specific version used would need to be chosen based on whether the cohort is actively exposed to $\mathrm{RHI}$ or is an older previously exposed group. 
A unique feature of the $\mathrm{RHICl}$ is the inclusion of both current values and rate of change from baseline in several elements. The $\mathrm{RHICl}$ is not intended to be used as a diagnostic test though a baseline score can be calculated to provide an anchor point from which clinical trajectory can be followed. Commonly used composite scales include measures that are thought to be characteristic of a particular condition and can differentiate patients from those without the disease. However, it is possible that the rate of change of some of these components may not be informative. By specifically assessing how certain tests change over time and integrating these elements into the $\mathrm{RHICl}$, we expect this instrument to be of particular value in monitoring longitudinal change or as an outcome measure in clinical trials.

The use of composite measures as endpoints in clinical trials have been encouraged by the Food and Drug Administration (FDA) and European Medical Agency (EMA) provided that the measures are carefully designed, are relevant to existing tools for which historical evidence exists, and are validated in independent prospective cohorts $(20,21)$. Given that the $\mathrm{RHICl}$ is based on data derived from commonly used neuropsychological tools, the first two requirements are met. However, the RHICl must be validated in separate samples. Unlike other neurodegenerative diseases such as Alzheimer's disease where large longitudinal datasets are publicly available, these types of data are more limited for those exposed to $\mathrm{RHI}$ (22). The PFBHS has over 100 additional participants who will be undergoing their 3rd time point assessment the next year; we will prospectively validate the $\mathrm{RHICl}$ on the larger sample as well as work with collaborators to validate the $\mathrm{RHICl}$ in other cohorts.

\section{Limitations}

In developing the $\mathrm{RHICl}$, we utilized all the cognitive and behavioral data collected from the PFBHS, a longitudinal observational study of active and professional fighters. However, it is important to acknowledge the limitations to this study. The extent of testing included several computerized cognitive batteries and self-administered behavioral inventories but was not exhaustive. The number of participants (particularly retired fighters and controls) who had complete data for at least 3 time points was limited. In regard to the latter, we would anticipate as more data become available from our cohort and others that the elements that comprise the $\mathrm{RHICl}$ may be modified or weighted differently. The computerized assessments used are proprietary, placing limitations on their widespread use.

\section{Conclusions}

There is an increasing need for tools to effectively track individuals exposed to RHI. With the potential of treating the consequences of $\mathrm{RHI}$ via clinical trials on the horizon, the proposed Repetitive Head Impact Composite Index, a sensitive measure to change in executive function, provides a standardized approach evaluating long-term effects of $\mathrm{RHI}$.

\section{Declarations}

\section{Ethics approval and consent to participate:}


The Professional Fighters Brain Health Study, from where the dataset for this study was obtained, was approved by the Cleveland Clinic Institutional Review Board and written informed consent was obtained from all participants.

\section{Consent for publication:}

Not applicable

\section{Availability of data and materials:}

The dataset used for analysis during the current study are available from the corresponding author on reasonable request

\section{Competing interests:}

CB has received research funding from the Ultimate Fighters Championship, Top Rank promotions, Haymon Boxing, Bellator/Spike TV

JLA has authored intellectual property related to the C3 Logix software

JC has provided consultation to Acadia, Actinogen, AgeneBio, Alkahest, Alzheon, Annovis, Avanir, Axsome, Biogen, Cassava, Cerecin, Cerevel, Cognoptix, Cortexyme, EIP Pharma, Eisai, Foresight, Gemvax, Green Valley, Grifols, Hisun, Karuna, MapLight, Novo Nordisk, Nutricia, Orion, Otsuka, ReMYND, Resverlogix, Roche, Samumed, Samus Therapeutics, Third Rock, Signant Health, Sunovion, Suven, and United Neuroscience pharmaceutical and assessment companies

\section{Funding:}

This study was made possible by financial support of the Professional Fighters Brain Health Study from: Ultimate Fighters Championship, Top Rank Promotions, Haymon Boxing, Bellator/Spike TV None of these funders had any role in the design of the study or collection, analysis, and interpretation of data, nor writing of the manuscript

\section{Authors contributions:}

CB, GS, LB, and JC were involved in design of study, analysis and interpretation of the results, and writing of manuscript

GS performed statistical analysis

JA was involved in instrument development and interpretation of results All authors read and approved the final manuscript 
Acknowledgments:

We would like to acknowledge the efforts of Pamela Dino who was essential to the conduct of the Professional Fighters Brain Health Study

\section{References}

1. Asken BM, Sullan MJ, DeKosky ST, Jaffe MS, Bauer RM. Research gaps and controversies in chronic traumatic encephalopathy: a review. JAMA Neurol. 2017;74:11256-1262.

2. McKee AC, Stein TD, Kiernan PT, Alvarez VE. The neuropathology of chronic traumatic encephalopathy. Brain Pathol. 2015 May;25(3):350-64.

3. Tharmaratnam T, Iskandar M, Tabobondung T, et al. Chronic Traumatic Encephalopathy in Professional American Football Players: Where are we now? Front Neurol. 2018;9:445. doi:10.3389/fneur.2018.00445.

4. Movement Disorder Society Task Force on Rating Scales for Parkinson's Disease. The Unified Parkinson's Disease Rating Scale: status and recommendations. Mov Disord. 2003;18(7):738-50. Doi:10.1002/mds.10473.

5. Wang J, Logovinsky V, Hendrix SB, et al. ADCOMS: a composite clinical outcome for prodromal Alzheimer's disease trials. J Neurol Neurosurg Psychiatry 2016: 87: 993-9. Doi. 10.1136/jnnp-2015$31 ` 2383$.

6. Goetz CG, Tilley BC, Shaftman SR, et al. Movement disorder society-sponsored revision of the unified Parkinsons disease rating scale: scale presentation and clinimetric testing results. Mov Disord 2008; 23: 2129-70. Doi10.1002/mds.22340.

7. Bernick C, Banks S, Phillips M, Lowe M, Shin W, Obuchowski N, Jones. Modic S. M.

8. Professional fighters brain. health study: rationale and methods. Am J Epidemiol. 2013;15:178:2806.

9. Gualtieri CT, Johnson LG. Reliability and validity of a computerized neurocognitive test battery, CNS Vital Signs [Comparative Study Research Support, Non-U.S. Gov't]. Arch Clin Neuropsychol. 2006;21(7):623-43.

10. Simon M, Maerlender A, Metzger K, Decoster L, Hollingworth A. Valovich McLeod T. Reliability and concurrent validity of select C3 Logix test components. Dev Neuropsychol. 2017;1-14.

11. Kroenke K, Spitzer R, Williams J. The PDQ - 9: Validity of a brief depression severity measure. J Gen Intern Med. 2001;16(9):606-13. Doi:10.1046/j.1525-1497.2001.016009606.x.

12. Potts $G$, George $M$, Martin L, et al. Reduced punishment sensitivity in neural systems of behavior monitoring impulsive individuals. Neurosci Lett. 2006;397:130-4.

13. McCoy C. Understanding the use of composite endpoints in clinical trials. West J Emerg Med. 2018;19:947-51. 
14. Goldberg R, Gore J, Barton B, et al. Individual and composite study endpoints: separating the wheat from the chaff. Am J Med. 2014;127:379-84.

15. International Conference on Harmonization of Technical Requirements for Registration of Pharmaceuticals for Human Use. ICH harmonized tripartite guideline: statistical principles for clinical trials. Stat Med. 1999; 18: 1905-1942.

16. Mendez MF. The neuropsychiatric aspects of boxing. Int J Psychiatry Med. 1995;249 - 62.

17. Bernick $C$, Banks $S$. What boxing tells us about repetitive head trauma and the brain. Alzheimers Res Ther. 2013;5:23. doi:10.1186/alzrt177.

18. Montenigro P, Baugh C, Daneshvar D, et al. Clinical subtypes of chronic traumatic encephalopathy: literature review and proposed research diagnostic criteria for traumatic encephalopathy syndrome. Alzheimer's Res Ther. 2014;6(5):68.

19. DeKosky ST, Blennow K, Ikonomovic MD, Gandy S. Acute and chronic traumatic encephalopathies: pathogenesis and biomarkers. Nat Rev Neurol. 2013;9(4):192-200.

20. Bernick $\mathrm{C}$, Shan $\mathrm{G}$, Zetterberg $\mathrm{H}$, et al. Longitudinal change in regional brain volumes with exposure to repetitive head trauma. Neurology. 2020;94:232-40. Doi:10.1212/WNL.000000000008817.

21. Food and Drug Administration. Early Alzheimer's disease: developing drugs for treatment. Guidance for Industry. 2018.

22. European Medicines Agency. Guideline on the clinical investigation of medicines for the treatment of Alzheimer's disease. 2018.

23. Weiner M, Veitch D, Aisen P, et al. 2014 Update of the Alzheimer's disease neuroimaging initiative: a review of papers published since its' inception. Alzheimer's Dement. 2015;11:e1-120. 